\title{
Process-property correlations in laser-induced graphene electrodes for electrochemical sensing
}

\author{
Arne Behrent $^{1}$ (D) $\cdot$ Christian Griesche $^{1}$ (D) $\cdot$ Paul Sippel $^{1} \cdot$ Antje J. Baeumner $^{1}$ (D)
}

Received: 20 November 2020 / Accepted: 18 March 2021

(C) The Author(s) 2021, corrected publication 2021

\begin{abstract}
Laser-induced graphene (LIG) has emerged as a promising electrode material for electrochemical point-of-care diagnostics. LIG offers a large specific surface area and excellent electron transfer at low-cost in a binder-free and rapid fabrication process that lends itself well to mass production outside of the cleanroom. Various LIG micromorphologies can be generated when altering the energy input parameters, and it was investigated here which impact this has on their electroanalytical characteristics and performance. Energy input is well controlled by the laser power, scribing speed, and laser pulse density. Once the threshold of required energy input is reached a broad spectrum of conditions leads to LIG with micromorphologies ranging from delicate irregular brush structures obtained at fast, high energy input, to smoother and more wall like albeit still porous materials. Only a fraction of these LIG structures provided high conductance which is required for appropriate electroanalytical performance. Here, it was found that low, frequent energy input provided the best electroanalytical material, i.e., low levels of power and speed in combination with high spatial pulse density. For example, the sensitivity for the reduction of $\mathrm{K}_{3}\left[\mathrm{Fe}(\mathrm{CN})_{6}\right]$ was increased almost 2 -fold by changing fabrication parameters from $60 \%$ power and $100 \%$ speed to $1 \%$ power and $10 \%$ speed. These general findings can be translated to any LIG fabrication process independent of devices used. The simple fabrication process of LIG electrodes, their good electroanalytical performance as demonstrated here with a variety of (bio)analytically relevant molecules including ascorbic acid, dopamine, uric acid, p-nitrophenol, and paracetamol, and possible application to biological samples make them ideal and inexpensive transducers for electrochemical (bio)sensors, with the potential to replace the screen-printed systems currently dominating in on-site sensors used.
\end{abstract}

Keywords Laser-induced graphene $\cdot$ Porous carbon $\cdot$ Fabrication parameters $\cdot$ Chemical sensor $\cdot$ Voltammetry

\section{Introduction}

Low-cost, distributed electrochemical sensors can be used to address challenges in many fields of modern life, such as environmental monitoring, industrial manufacturing, and food production, and-most prominently-healthcare. Exemplary electrochemical biosensors for the measurement of blood glucose concentration have matured through decades of research and were successfully commercialized by several companies $[1,2]$. These small disposable electrode strip sensors combined with a handheld glucose meter and supplied with a few microliters of blood from a pin-prick

Antje J. Baeumner

antje.baeumner@ur.de

1 Institute of Analytical Chemistry, Chemo- and Biosensors, University of Regensburg, Regensburg, Germany enable now millions of diabetes patients to manage their daily lives. Motivated by this success story, researchers hope to enable users to measure many other chemical parameters related to health, mostly applied to diseases where the patients benefit from continuous or frequent monitoring. While the proposed sensors naturally feature different details in their detection mechanism to provide suitable sensitivity and specificity for a given application, many rely on the same concept of electrochemical detection with a disposable, modified electrode, which means electrode materials as well as the production process need to be reliable and inexpensive. At the moment, the most common commercial electrode type is the screen-printed carbon electrode [3-5]. However, in most recent years, among the many nanomaterials proposed as better electrode material, laserinduced graphene (LIG) was described which has the potential to truly challenge and eventually substitute screenprinted electrodes (SPEs) $[6,7]$. 
LIG is a porous graphene-like material that can be created by simply pointing a sufficiently strong $\mathrm{CO}_{2}$ laser onto commercial polyimide foil (e.g., Kapton ${ }^{\circledR}$ ) in an ambient environment. The exact mechanism of the conversion has not been fully described and is also not the topic of this publication, but the following can be assumed to take place. A strong focused laser beam with a pulse length on the order of microseconds locally heats the substrate to temperatures above $2500{ }^{\circ} \mathrm{C}$ [8] followed by rapid cooling. At least part of the irradiated polymer immediately melts/evaporates, causing the porous nanoand microstructure observable by scanning electron microscopy (SEM). Photothermal [8] bond breaking rearranges the structure into mostly $\mathrm{sp}^{2}$-carbons while the carbon content increases from below 70 to above $90 \%$, as non-carbon elements partly remain in the material, possibly incorporated as heteroatoms in the dominating hexagonal carbon rings, but mostly evaporate as gases. Carbonization is readily confirmed by a color change from orange to black. Examination of the created material via transmission electron microscopy (TEM), X-ray diffraction measurement (XRD), and Raman spectroscopy further reveal high similarity to few-layer graphene, confirming the $\mathrm{sp}^{2}$ carbon structures and giving rise to the name laser-induced graphene [6, 9].

With a commercial computer-controlled laser cutter, one can create two-dimensional patterns, a process generally termed direct laser writing (DLW). The obtained LIG patterns can have a spatial resolution of roughly 25 to $150 \mu \mathrm{m}$, depending on the employed focusing lens, and can be used as electrical transducers for various applications. DLW on Kapton film was first reported by the Tour group at Rice University in 2014 which also coined the term LIG and emphasized its possible application for the production of microsupercapacitors [6]. In subsequent publications, they reported areal capacitances of $4-16.5 \mathrm{mF} / \mathrm{cm}^{2}$ for pristine and heteroatom-doped LIG, which could be raised to $934 \mathrm{mF} / \mathrm{cm}^{2}$ by deposition of $\mathrm{MnO}_{2}$ for additional pseudocapacitance [10]. Since then, the material has been further characterized and variations of the manufacturing process have been explored by choosing different substrates - among them wood, paper, and even coconut shells - or by changing the composition of the lasing atmosphere $[11,12]$. Much of this has been covered in two recent reviews published by the Tour group [9, 13].

Since LIG is a graphene-like pure carbon material that can be made inexpensively in any chosen two-dimensional shape, it seems an obvious candidate for disposable electrodes used in electrochemical sensing, an area, which is dominated by screen-printed carbon electrodes (SPCE) at the time. The largest existing market for disposable biosensors is blood glucose monitoring for diabetes patients and commercial electrodes used in blood glucose sensing are made by screen-printing, an inexpensive method suitable for mass production. In this method, a suspension of conductive material is transferred onto a support through a fine mesh, except in those places covered by a stencil, defining the desired shape of electrodes, leads, and contacts. Afterwards, the pattern is baked to solidify the suspension. With LIG electrodes, on the other hand, no material other than the polyimide foils is needed, and no baking step is necessary; the pattern is created without the need for any additional binder substances and can thus easily withstand most organic solvents. Furthermore, less material is wasted in this process and a change in pattern design is easily realized as it only requires a new drawing on the computer. In contrast, stencils must be prepared for each design change in the SPCE printing process. These differences suggest that LIG electrodes have a high potential to become a preferable electrochemical transducer for point-of-care applications as their production ought to be simpler, less expensive, and easily adaptable to a roll-to-roll fabrication process. We and others have employed LIG for electrochemical sensors, e.g., Nayak et al. have shown that the oxidation peaks for the biological analytes dopamine, ascorbic acid, and uric acid could be well resolved in pulsed voltammetry on LIG [14]. Fenzl et al. used pyrenebutyric acid to couple a thrombin aptamer to the LIG matrix and demonstrated that it can be successfully used in an aptasensor [15]. Also, recent work from our lab describes a combination of amperometry-, potentiometry-, and impedance-based sensors made from LIG for the measurement of lactate, potassium, and conductivity in sweat [16]. Other groups have modified DLW-created carbon electrodes with copper nanoparticles alone or in combination with diamine oxidase to detect glucose or biogenic amines respectively $[17,18]$. The group of Claussen has used LIG to make ion-selective electrodes for the detection of ammonium and nitrate in soil [19] or ammonium and potassium in human urine [20]. Cardoso et al. and Beduk et al. reported the successful use of molecularly imprinted polymers (MIP) on LIG electrodes to detect chloramphenicol and bisphenol A, respectively $[21,22]$. More examples for the use of this material in electrochemical biosensing are listed in recent review papers by Kurra et al. [7] and Lahcen et al. [23].

Through all of these studies, it is generally recognized that the laser power, the laser beam size at the substrate surface, the speed at which the laser beam spot progresses during writing (i.e., the scan speed, $v$ ), and the spatial laser pulse density (i.e., how many times the laser fires per distance traveled over the substrate) all influence the procedural outcome. That is, they give rise to different structures in the nano- and micrometer regime, as well as differences in the Raman spectrum, electrical conductivity, and hydrophilicity of the interface. We postulated, therefore, that these parameters will have a significant impact on LIG's usability as electroanalytical transducer since electroanalytical performance is mainly determined by conductance, electron transfer, and electrode surface area. Here, we report on a detailed study investigating the performance of LIG electrodes fabricated over a large parameter space of laser power, scan speed, and different pulse densities in an effort to 
provide a systematic evaluation and understanding of how LIG fabrication parameters influence performance in electrochemical sensing. The reported observations were made with a specific commercial flatbed laser engraving system but can be translated to other machines that operate in a similar manner. The findings will also support future work on binder-free carbon electrode materials and help elucidate the relative importance of LIG characteristics on actual electroanalytical performance in real-world settings.

\section{Materials and methods}

\section{Chemicals}

Potassium ferricyanide $\left(\mathrm{K}_{3}\left[\mathrm{Fe}(\mathrm{CN})_{6}\right]\right)$, potassium ferrocyanide $\left(\mathrm{K}_{4}\left[\mathrm{Fe}(\mathrm{CN})_{6}\right]\right)$, hexaammineruthenium trichloride $\left(\left[\mathrm{Ru}\left(\mathrm{NH}_{3}\right)_{6}\right] \mathrm{Cl}_{3}\right)$, dopamine hydrochloride, uric acid, Lascorbic acid, p-nitrophenol, acetaminophen, and methylene blue were obtained from Sigma Aldrich in analytical grade. Chemicals needed for preparation of phosphate-buffered saline (PBS, $8.1 \mathrm{mM} \mathrm{Na}_{2} \mathrm{HPO}_{4}, 1.9 \mathrm{mM} \mathrm{KH}_{2} \mathrm{PO}_{4}, 2.7 \mathrm{mM} \mathrm{KCl}$, $137 \mathrm{mM} \mathrm{NaCl}, \mathrm{pH}$ 7.4) were bought from Merck (Germany) or Carl Roth (Germany) in analytical grade. All chemicals were used without further purification. Non-adhesive Kapton $\mathrm{HN}$ foil $(125 \mu \mathrm{m})$ was bought from CMC Klebetechnik $\mathrm{GmbH}$ (Frankenthal/Pfalz, Germany), and conductive silver paint was obtained from Busch $\mathrm{GmbH} \&$ Co. KG. (Viernheim, Germany).

\section{Electrode fabrication}

Electrode patterns were designed on a computer with the vector graphics software CorelDraw. A model VLS2.30 laser cutter (Universal Laser Systems, Scottsdale, AZ, USA) equipped with a $30 \mathrm{~W} \mathrm{CO}_{2}$-laser $(10.6 \mu \mathrm{m})$ and focused beam diameter of approx. $125 \mu \mathrm{m}$ ( 2 " lens) was used to create LIG electrodes on Kapton foil. The foil was cut into sheets of suitable size and the borders were fixed with tape directly onto the machine's engraving table which was brought into focal distance. The $z$-distance yielding the smallest laser spot mark on a test material was regarded as the accurate focal distance with an absolute value of an estimated $5.1 \pm 0.1 \mathrm{~cm}$. A fabrication scheme and two examples for electrode designs are shown in Fig. 1.

The power setting ( 1 to $100 \%$ of $30 \mathrm{~W}$ ), movement speed of the lens carriage in $x$-direction ( $1 \%$ to $100 \%$ of 50 inches/s), and spatial laser pulse density (with the fixed combinations of 500 by 500,1000 by 1000 , or 1000 by 2000 PPI (pulses per inch in $x$ - and $y$-direction)) were varied using the machine software. Percent values are used here instead of physical units to report power and speed settings (e.g., "1\% power" instead of " $0.3 \mathrm{~W}$ ") because \% power and measured power scale linearly only approximately so that a direct translation into physical units may in some cases be imprecise. For brevity, the following shorthand was adopted for scribing conditions, in which e.g., " $1 / 10 / 1000 \times 2000$ " means " $1 \%$ power, $10 \%$ speed, 1000 PPI in $x$-direction, and 2000 PPI in $y$-direction." The distinction between $x$ - and $y$-directions, indicated in Fig. 1a, is meaningful because the lens carrier will only travel quickly in the former and more slowly in the latter direction, which is inevitable due to the way the positioning system is constructed, as the laser moves with the set speed and frequency along the $x$-axis followed by a move in the $y$-direction to then continue the scan at the set speed and frequency back along the $x$-axis. Nonetheless, the device delivers the desired PPI in each direction. Air was extracted from the scribing chamber continuously during operation to remove soot and any emerging gases.

Pure argon and nitrogen gas could be supplied directly to the processing chamber to modulate the atmosphere. The gases were released directly at the lasing spot through the airassist cone (see Fig. S1 in Online Resource 1). However, in contrast to the experiments with controlled-atmosphere chambers done by $\mathrm{Li}$ [12] and others, the supplied gases were inevitably diluted with air that was continually sucked into the

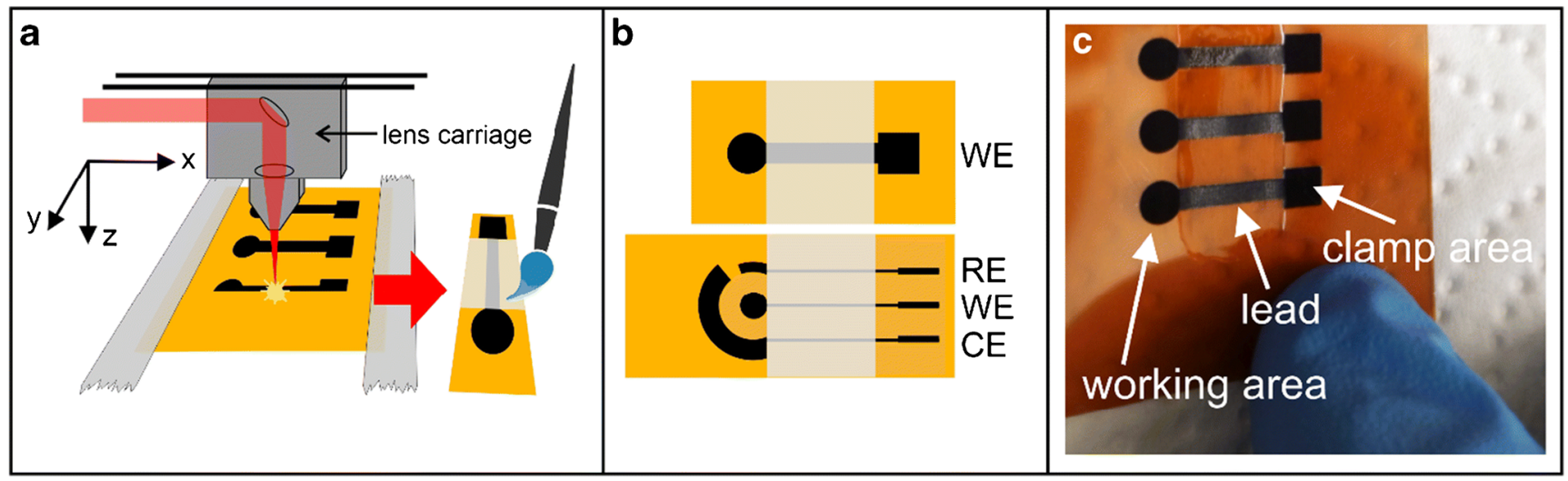

Fig. 1 Electrode fabrication scheme (a), sketches of used electrode designs (WE working electrode, CE counter electrode, RE reference electrode), top = "simple" design, bottom = 3-electrode design $(\mathbf{b})$, photo of prepared LIG electrodes $(\mathbf{c})$ 
(non-airtight) scribing chamber by the extraction system. Therefore, the lasing atmosphere was enriched in Ar or $\mathrm{N}_{2}$ but measured data on gas composition is not available.

After scribing, electrodes were rinsed with water, then isopropanol, and then dried under a nitrogen stream. Transparent nail polish was painted over the leads to restricting contact with electrolyte solution only to the electrode area. Post-scribing steps can be viewed in the supplied video files (Online Resources 2, 3, 4, 5). Electrodes were stored in non-airtight boxes at room temperature in the lab, until used.

\section{Electrochemical analysis}

For all electrochemical measurements, the analyte was dissolved in $1 \times$ PBS ( $\mathrm{pH} 7.4$ ). A PalmSens 4 potentiostat (PalmSens BV, Netherlands) was used. For screening measurements, in which the same electrolyte could be reused many times, the simpler electrode design version of LIG (Fig. 1b, top) was clamped as working electrode (WE) and dipped into approx. $10 \mathrm{~mL}$ of electrolyte together with a platinum wire as a counterelectrode (CE) and a pole $\mathrm{Ag} / \mathrm{AgCl}$ reference electrode (RE) (BAS Inc., USA). On the other hand, the 3-electrode design (Fig. 1b, bottom) was more convenient for the recording of calibration plots and working with small volumes of electrolytes. Here, a $50-\mu \mathrm{L}$ droplet was placed onto the $\mathrm{LIG}$ electrode (WE and $\mathrm{CE}$ ) and a $\mathrm{Ag} / \mathrm{AgCl}$ reference electrode was contacted from above. The small LIG electrode in the 3-electrode design was intended as the basis for a future reference electrode but not used in these investigations. Figure S2 shows photos of both cell arrangements. Voltammetric peak potentials and heights were detected either fully- or semi-automatically through the software PSTrace 5.8 with a linear baseline. For the fabrication parameter survey, cyclic voltammetry $(\mathrm{CV})$ of $5 \mathrm{mM} \mathrm{K}_{3}\left[\mathrm{Fe}(\mathrm{CN})_{6}\right]$ in PBS + $0.1 \mathrm{M} \mathrm{KCl}(\mathrm{pH} \mathrm{7.4)}$ was carried out with a scan rate of $50 \mathrm{mV} / \mathrm{s}$ at $1 \mathrm{mV}$ steps and the peak-to-peak distance was calculated as $\Delta E_{\mathrm{p}}=E_{\mathrm{p}, \mathrm{ox}}-E_{\mathrm{p}, \text { red }}$ to serve as a measure of electrode quality. $\mathrm{CV}$ with scan rates between 25 and $200 \mathrm{mV} \mathrm{s}^{-1}$ was then recorded for a selected electrode type to determine its electrochemically active surface area (ESA) and effective heterogeneous electron transfer rate for $\left(k^{0, \text { eff }}\right)$, as described in the supplementary information. Square-wave voltammetry was used for quantitative analysis and run at $5 \mathrm{mV}$ step, $50 \mathrm{mV}$ amplitude, and a frequency of $10 \mathrm{~Hz}$.

\section{Sheet resistance}

The electrical sheet resistance was measured with a fourpoint-probe apparatus built in-house: four spring-loaded golden pins, arranged in a straight line and spaced equally by $1.5 \mathrm{~mm}$ were used to contact the center of a $1-\times 1-\mathrm{cm}$ LIG sample. The probing current was $100 \mu \mathrm{A}$.

\section{Physical characterization}

Infrared reflectance spectra were recorded over the range of 4000 to $650 \mathrm{~cm}^{-1}\left(8 \mathrm{~cm}^{-1}\right.$ resolution) on an Agilent Cary630 equipped with a diamond single bounce attenuated-totalreflectance attachment. 32 scans were averaged for sample and background each. The elemental composition of Kapton and LIG was measured via combustion analysis on a Vario Micro Cube. Raman spectra were collected from 50 to $3500 \mathrm{~cm}^{-1}$ on a Thermo Fisher DXR Raman microscope with a $532-\mathrm{nm}$ laser set to $8-\mathrm{mW}$ power and a $\times 50$ objective with an estimated focal spot diameter of $0.7 \mu \mathrm{m} .16$ scans were averaged per spot.

X-ray photoelectron spectroscopy (XPS) studies were carried out in a Kratos Axis Supra DLD spectrometer equipped with a monochromatic $\mathrm{Al} \mathrm{K} \alpha \mathrm{X}$-ray source $(h \nu=1486.6 \mathrm{eV})$ operating at $150 \mathrm{~W}$ and a vacuum of $\sim 10^{-9}$ mbar. Survey and high-resolution spectra were collected with pass energies of $160 \mathrm{eV}$ and $20 \mathrm{eV}$ respectively. Samples were mounted in floating mode in order to avoid differential charging. Charge neutralization was required for all samples. Binding energies were referenced to the $\mathrm{sp} 2$ hybridized $(\mathrm{C}=\mathrm{C})$ carbon for the $\mathrm{C}$ $1 \mathrm{~s}$ peak set at $284.4 \mathrm{eV}$ from graphene.

SEM images were obtained with a Zeiss LEO 1530. Water contact angles were recorded with the sessile drop method on an OCA 25 system with corresponding software (DataPhysics Instruments GmbH, Filderstadt, Germany).

\section{Results and discussion}

Laser-induced graphene was studied as an alternative, carbonaceous material for electrochemical (bio)sensing applications. Its promising characteristics described by us earlier $[15,16]$ indicate that it may be not only an alternative but a superior transducer material for electrochemical point-of-care sensors. Not much is known about the effect of energy input during the fabrication in correlation to electroanalytical performance, only anecdotal data are available describing obtainable micromorphologies. By systematically studying process parameters that influence the energy input and correlating it to micromorphologies, Raman, and especially electroanalytical characteristics, this knowledge gap was sought to be filled by the study.

\section{Understanding of scribing parameters' effects on laser-induced graphene}

We created LIG electrodes with a circular working area $(d=$ $3 \mathrm{~mm}, A=0.071 \mathrm{~cm}^{2}$ ) connected via a bridge to an electrical contact area for electrochemical testing (see Fig. 1c). To assess the influence of available laser instrument parameters, the power- and speed settings as well as spatial pulse density were systematically varied. 
The electrodes were judged by mechanical integrity and visual appearance, represented by the heatmap overview for a medium pulse density setting (Fig. 2). Representative different visual appearances are shown in the inset. Electrodes of homogenous texture that would withstand bending without delamination were created by a suitable combination of power and speed (area of green color in Fig. 2). On the contrary, too much energy input resulted in brittle electrodes that would peel off the substrate easily. This was the case when the power setting was not ideal and hence a bit too high or the speed a bit too low (red and orange color). At low power and high-speed settings, the substrate was not carbonized or only partially (brown colors). When the energy input was just at the lower limit necessary for carbonization, only part of the desired shape was carbonized and instead triangular shapes appeared (see the second electrode from bottom in the inset of Fig. 2). This odd phenomenon stems from the carbonization starting at randomly located but energetically favorable nucleation points on the substrate. Those initially carbonized islands then promote carbonization in the immediate vicinity through increased absorbance which causes the lines of converted LIG to become longer with each consecutive sweep and create the observed triangular patterns with the tip facing upwards. The tips face downwards when the laser scanning direction is reversed (i.e., going from bottom to top regarding the $y$-direction).

While all green-marked laser settings generated visually similar electrodes, conductivity, and electrochemical activity differed. Cyclic voltammograms (CVs) were recorded on a series of electrodes in whose fabrication all parameters but one were kept constant to demonstrate the influence of a single parameter. The settings of $1000 \times 1000$ PPI and a constant power of $30 \%$ were chosen, because at this point, the speed could be varied on a wide range while producting functional electrodes (see Fig. 2). The resulting CVs in Fig. 3a show that peak-to-peak separation $\left(\Delta E_{\mathrm{p}}\right.$, Fig. 3b) and sheet resistance (Fig. 3c) both increase with scribing speed. To remove the influence of lead resistance from CV analysis, the leads were painted with conductive silver paste. Consequently, $\Delta E_{\mathrm{p}}$ values dropped overall and were influenced less by the scribing speed. Apparently, the high resistance of the LIG leads causes significant potential drop (iR-drop) between electrode working area and connecting clamp, which distorts the shape of the voltammograms. Longer leads caused higher drop (resistivity factor) but also larger electrode surfaces, higher concentration of redox species, or increased scanning speed in potential sweeping experiments (current factor). Strategies to prevent iR-drop therefore include the use of small electrodes and low redox species concentrations, if application of conductive paint is to be avoided. The lead dimensions of a designed electrode are usually dictated by practical reasons and therefore offer little room for adjustment. However, with optimized laser settings (see below), the sheet resistance of LIG could be reduced to as low as $10-20 \Omega$ sq. $^{-1}$ which greatly reduced the iR-drop problem (the value of 10-20 $\Omega \mathrm{sq}^{-1}$ was not a specific target, but it was the best achievable). Therefore,
Fig. 2 Heatmap of electrode outcome vs. power and speed settings at a pulse density of $1000 \times 1000(x$ by $y)$ (color code as indicated in the inset: green $=$ ok, darker brown $=$ partial scribing (PS), lighter brown $=$ no effect $(\mathrm{NE})$, orange $=$ LIG peeled off from substrate $(\mathrm{PO})$, red $=$ laser burned through substrate (B))

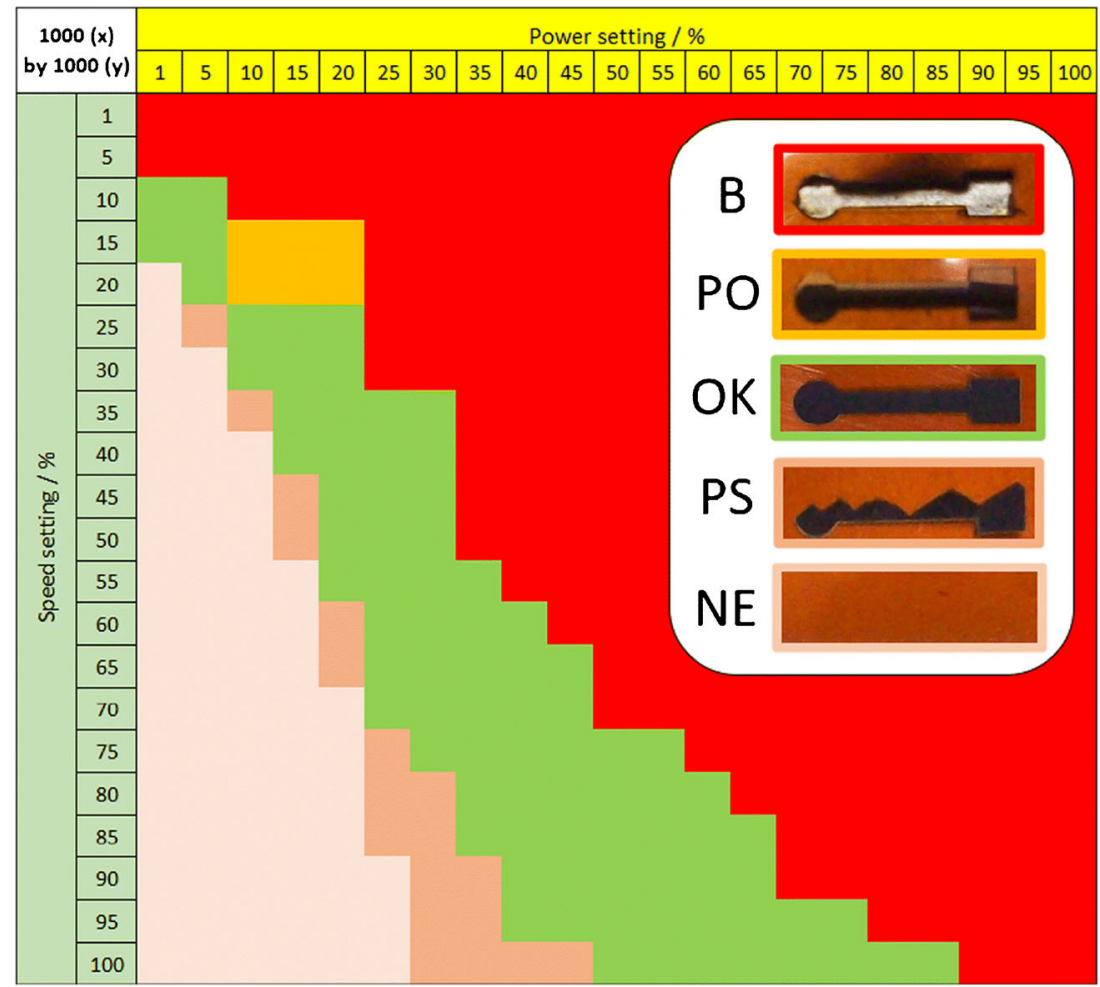



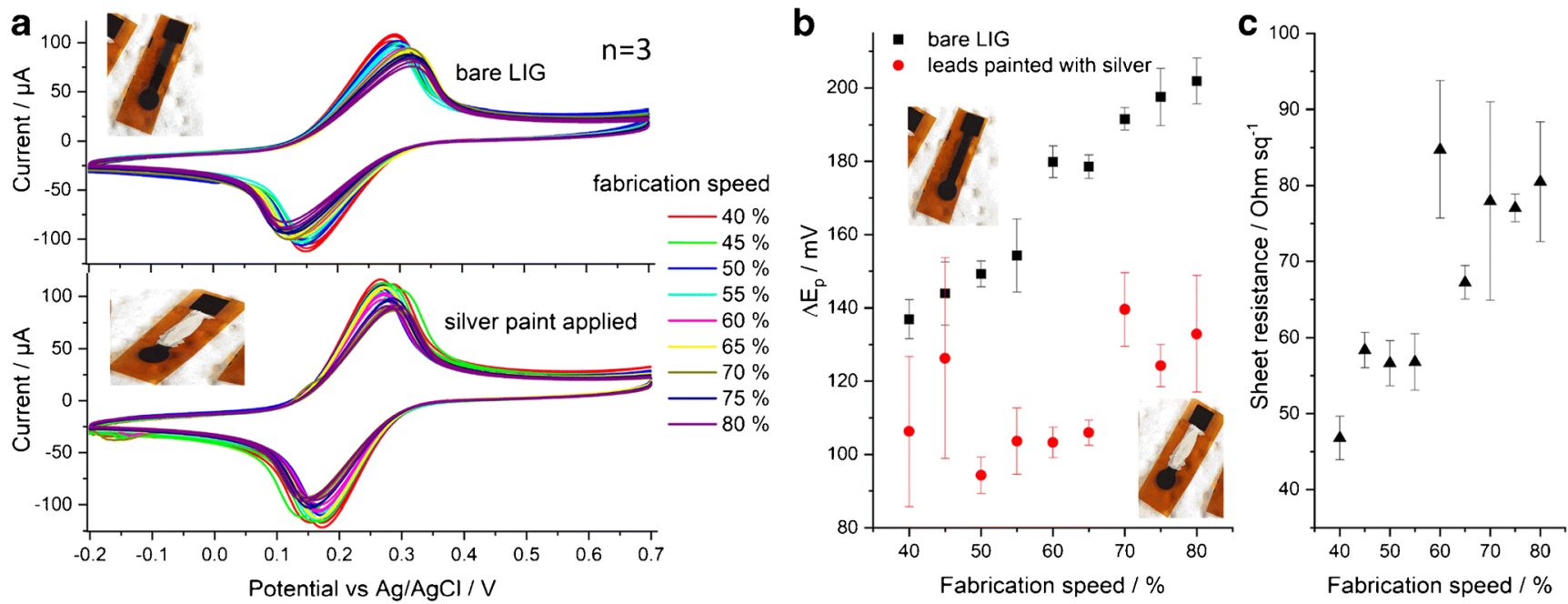

Fig. 3 a CV of $5 \mathrm{mM} \mathrm{K}_{3}\left[\mathrm{Fe}(\mathrm{CN})_{6}\right]$ in PBS with electrode leads bare or covered with silver paint. Higher speed setting correlated with larger $\Delta E_{\mathrm{p}}($ laser power $=30 \%, 1000 \times 1000$ PPI). b Peak-to-peak separation in CV. $\mathbf{c}$ sheet resistance of LIG vs scribing speed

it was possible to make an all-LIG electrode without additional processing steps, like painting the leads, when the right scribing conditions are used.

Furthermore, the influence of laser pulse density was investigated. The laser cutter used in this study permitted only certain locked pulse density settings. Hence, $500 \times 500$ PPI, $1000 \times$ 1000 PPI, and $1000 \times 2000$ PPI were compared $(x$ - by $y$-direction). The heatmap in Fig. 2 is collected at $1000 \times 1000$ PPI which resulted in a particularly good set of electrodes at a broader range of settings. Some power/speed combinations at the higher pulse density of $1000 \times 2000$ PPI yielded even better conductivity and probably electron transfer. However, these electrodes were sometimes prone to delamination and the design-space for power and speed was narrower at the increased pulse density (see Fig. S3). Therefore, the medium setting of $1000 \times 1000$ PPI was used in this work to create LIG electrodes for sensing applications. Furthermore, in terms of output, scribing the same pattern at 2000 PPI in $y$-direction takes twice as long compared to 1000 PPI. Power/speed heatmaps recorded at other pulse density settings can be found in Fig. S3 along with a comparison of CV performance among the best electrodes obtained at different pulse density settings in Fig. S4. Electrodes created with the lowest spatial pulse density option of $500 \times 500$ PPI ranked lowest in conductivity and were deemed less useful for electrochemical measurements.

It should be mentioned that the exact results of Fig. 2 (and the other maps) are likely not directly translatable to machines with a different maximum laser power or different size of focused beam. Even the size of the desired pattern or placing the substrate too close to the limit of the scribing area can influence the carbonization outcome. However, from experience with different flatbed laser systems, we have found that optimal power/speed conditions always follow more or less the green diagonal region in Fig. 2. Specifically, as a rule of thumb, it seems that low power, low speed, and high spatial pulse density will create LIG electrodes with high conductivity and generally good electron transfer behavior at the electrode-electrolyte interface.

Based on these findings, the setting of $1 \%$ power, $10 \%$ speed, and $1000 \times 1000$ PPI $(1 / 10 / 1000 \times 1000)$ was selected as a very conductive, electrochemically active, and mechanically sturdy LIG material. Unless different settings are specifically mentioned, these settings were used to produce electrodes for all following electrochemical tests, and scribing took about $1 \mathrm{~min}$ per electrode. It should be pointed out though that many of the settings within the green range of the heat map (Fig. 2) can be suitable for a user's electroanalytical needs and be created at higher throughput.

To demonstrate the variation in chemical sensing performance within the same pulse density setting, three electrode types made with increasing energy input were chosen from the green area in Fig. 2: 1/10, 25/40, and 60/100 (the first number denotes \%power, the second \%speed), and SWV responses were recorded to standards of $\left[\mathrm{Fe}(\mathrm{CN})_{6}\right]^{3-}$ in concentrations between 1 and $100 \mu \mathrm{M}$ (Fig. 4). The sensitivity (see Fig. S16) decreases from type 1/10 over $25 / 40$ to $60 / 100$ which correlates with rising sheet resistance of the different LIG types: $(26 \pm 0.6) \Omega$, $(49 \pm 1.5) \Omega$, $(57 \pm 6) \Omega$ for $1 / 10,25 / 40$, and $60 / 100$, respectively. While all three electrode types appeared visually homogenous, the microstructure of $1 / 10$ appears most uniform and flat while 25/40 shows fibrous structures of LIG and gaps. This appearance is even more pronounced in LIG type 60/100.

Fig. 4 Micrographs showing the active region of LIG electrodes made with the same pulse density but different power and speed settings (a, d, g). Square-wave voltammograms $(\mathbf{b}, \mathbf{e}, \mathbf{h})$ and dose-response plots $(\mathbf{c}, \mathbf{f}, \mathbf{i})$ of ferricyanide standards on those electrodes. $E_{\text {begin }}=0.7 \mathrm{~V}, E_{\text {end }}=$ $-0.2 \mathrm{~V}$, step $=5 \mathrm{mV}$, pulse $=50 \mathrm{mV}, f=10 \mathrm{~Hz}$. All standards $(\mathrm{V}=$ $50 \mu \mathrm{L}$ ) were measured on the same electrode in order of rising concentration. 3-electrode design LIG electrodes were used 

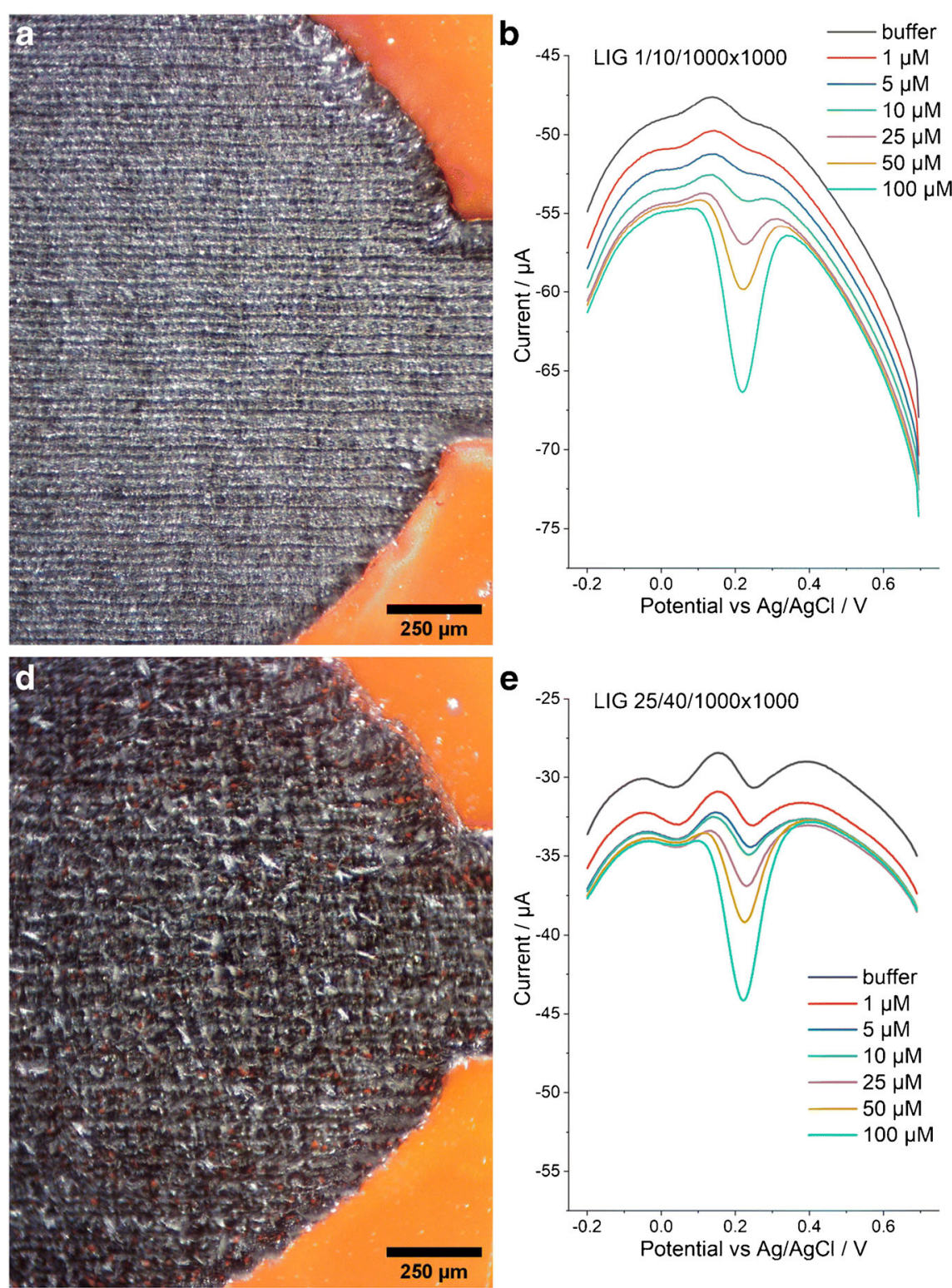

e
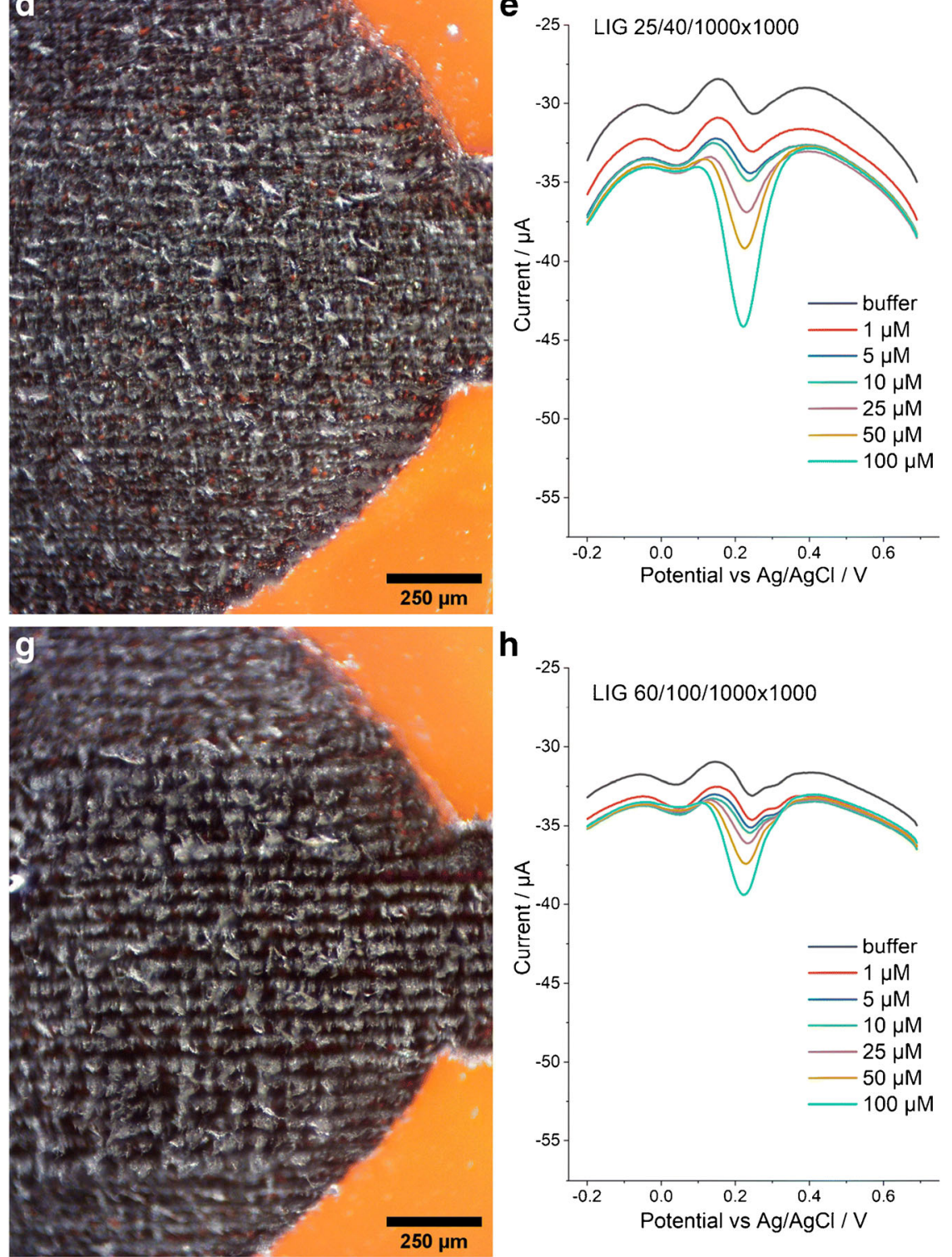

c

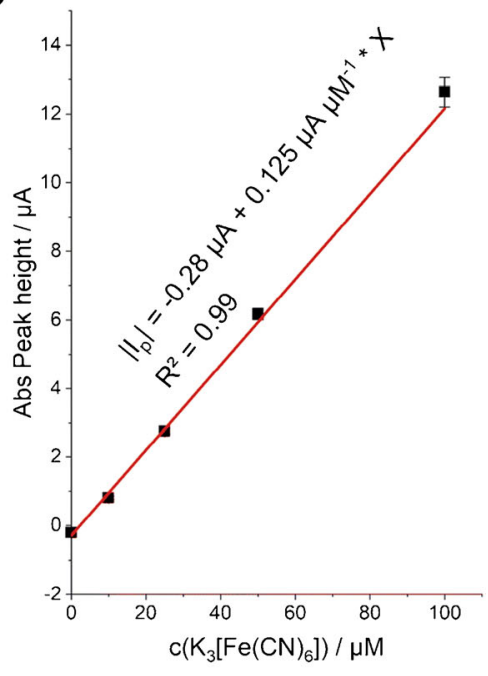

f

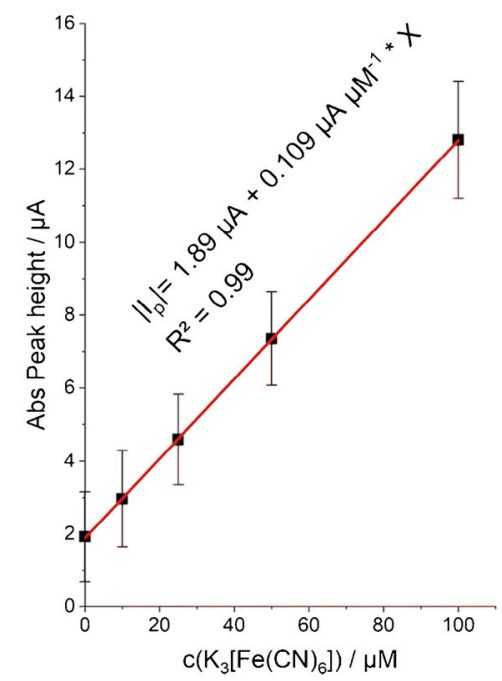

i

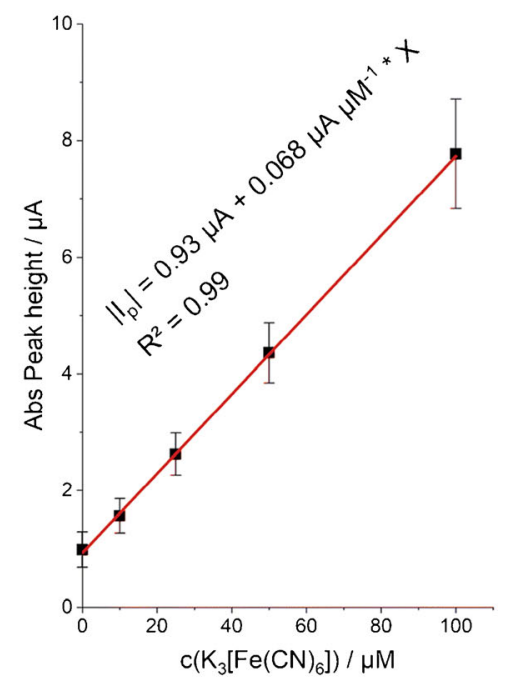


One concern about electrode manufacturing reproducibility regarded the location of the polyimide substrate in the machine when being scribed, since the lens carrier might be slower in some regions than in others due to available acceleration distance. Electrodes were thus scribed in several relative locations on the engraving table and peak-to-peak distances were measured. Also, $1-\times 1-\mathrm{cm}$ squares of LIG were made in the same locations to measure the sheet resistance. It was found that substrate location had no significant impact on performance in CV (Fig. 5a), measured as peak-to-peak separation. All electrodes showed around $100-\mathrm{mV}$ peak-to-peak separation but the - overall low - sheet resistance values dropped from $33 \Omega$ at the top left location to $23 \Omega$ in the bottom right position (Fig. $5 b$ ). We conclude that while the effect of location on sheet resistance may be kept in mind, for most electrochemical experiments, no special attention needs to be paid as to where the substrate is placed during electrode manufacture.

It is known that the presence of doping atoms such as nitrogen can increase the overall conductance of graphenelike materials [24]. It was therefore investigated if such doping could be achieved by simply changing the gas environment during the scribing process. Therefore, the laser cutter was flooded through a port in the chamber with either nitrogen or argon during operation. While this simple approach admittedly did not create a perfect gas atmosphere, it can quickly be realized in any lab. The LIG surfaces prepared under argon and nitrogen became very hydrophobic with water contact angles of $170^{\circ}$ and $150^{\circ}$ respectively, while the sessile drop spread completely on LIG prepared under ambient atmosphere. The gas environment influenced the microstructure (Fig. S9) and also the Raman spectrum (Fig. S10) but oddly seemed not to cause significantly different outcomes in cyclic voltammetry (Fig. S11). In terms of large-scale fabrication, this indicates that the ambient atmosphere is sufficient to produce high-quality electrodes. More in-depth investigations regarding the influence of lasing atmosphere on spectral characteristics and hydrophobicity of LIG were published by Li et al. and Mamleyev et al. [12, 25]

\section{Physicochemical characterization}

Infrared reflectance spectra of the Kapton substrate and LIG are displayed in Fig. S5A. The features of polyimide in the fingerprint region between 600 and $1800 \mathrm{~cm}^{-1}$ completely disappeared and the overall transmission dropped profoundly across the whole spectrum after carbonization. Both observations confirm the chemical transformation to carbonaceous LIG.

The carbon content increased from roughly $68 \%$ in Kapton to above $93 \%$ in LIG (Fig. S5B), indicative of carbonization, while contents of hydrogen and nitrogen decreased from 3 and $7 \%$ to values below $1 \%$, likely being released as gases during the scribing process. The oxygen content dropped to $5 \%$ after scribing, which points toward the presence of oxygencontaining groups in the carbon lattice of LIG, which were also found via XPS analysis (Fig. S7). The Raman spectrum of LIG features the characteristic $D, G$, and 2D peaks known from graphene-like materials (Fig. S6).

SEM micrographs of porous LIG of the type 1/10/1000 $\times$ 1000 are shown in Fig. 6. A pattern of horizontal trenches is visible at low magnification (Fig. 6b), which was created when the pulsed laser beam passed over the substrate in successive lines from top to bottom with a pitch of about $25 \mu \mathrm{m}$. Given this pitch and the beam diameter of approximately $125 \mu \mathrm{m}$, each location on the surface specified by the pattern
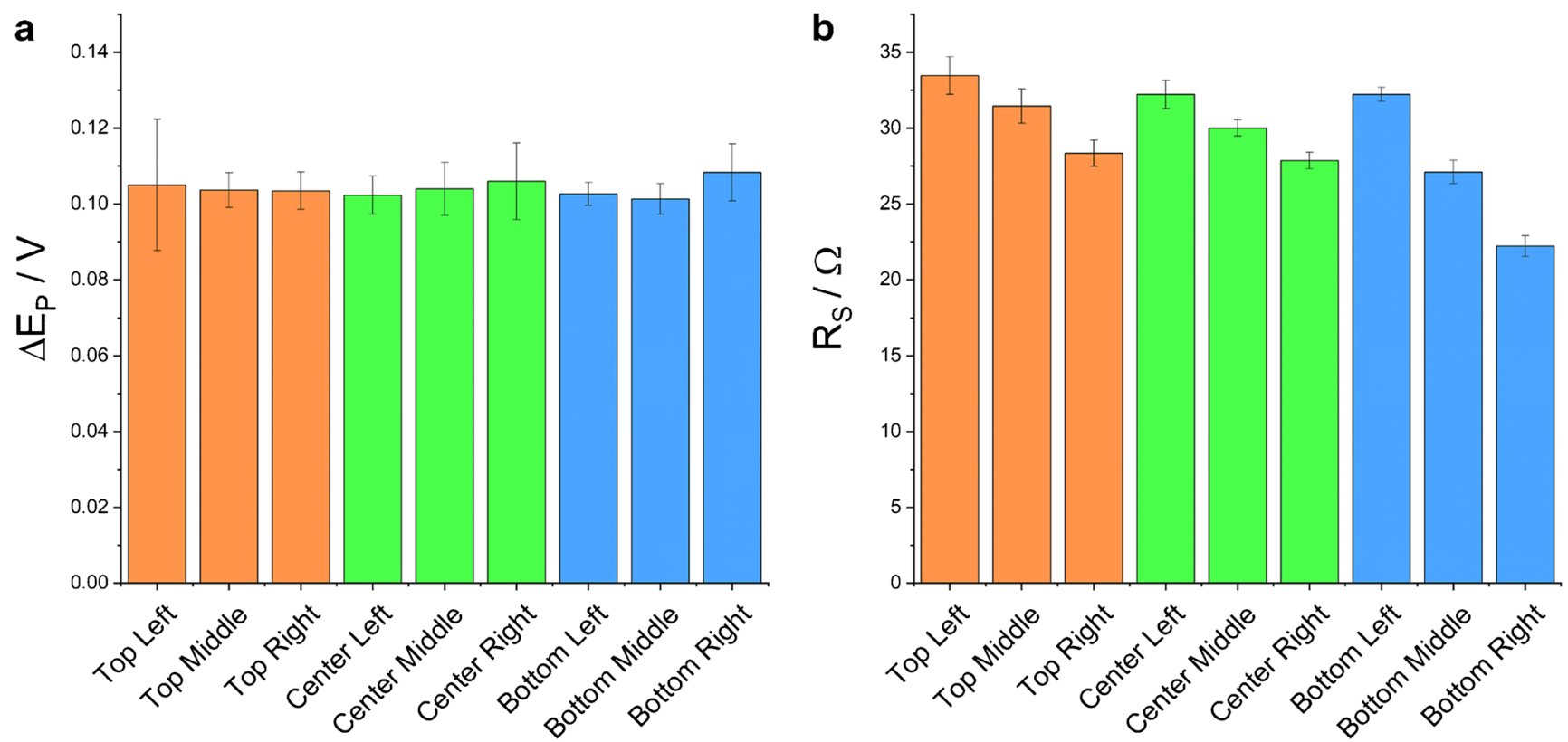

Fig. 5 Peak-to-peak distance obtained with LIG electrodes in CV (a) and sheet resistance of LIG samples $(\mathbf{b})(n=3)$ 

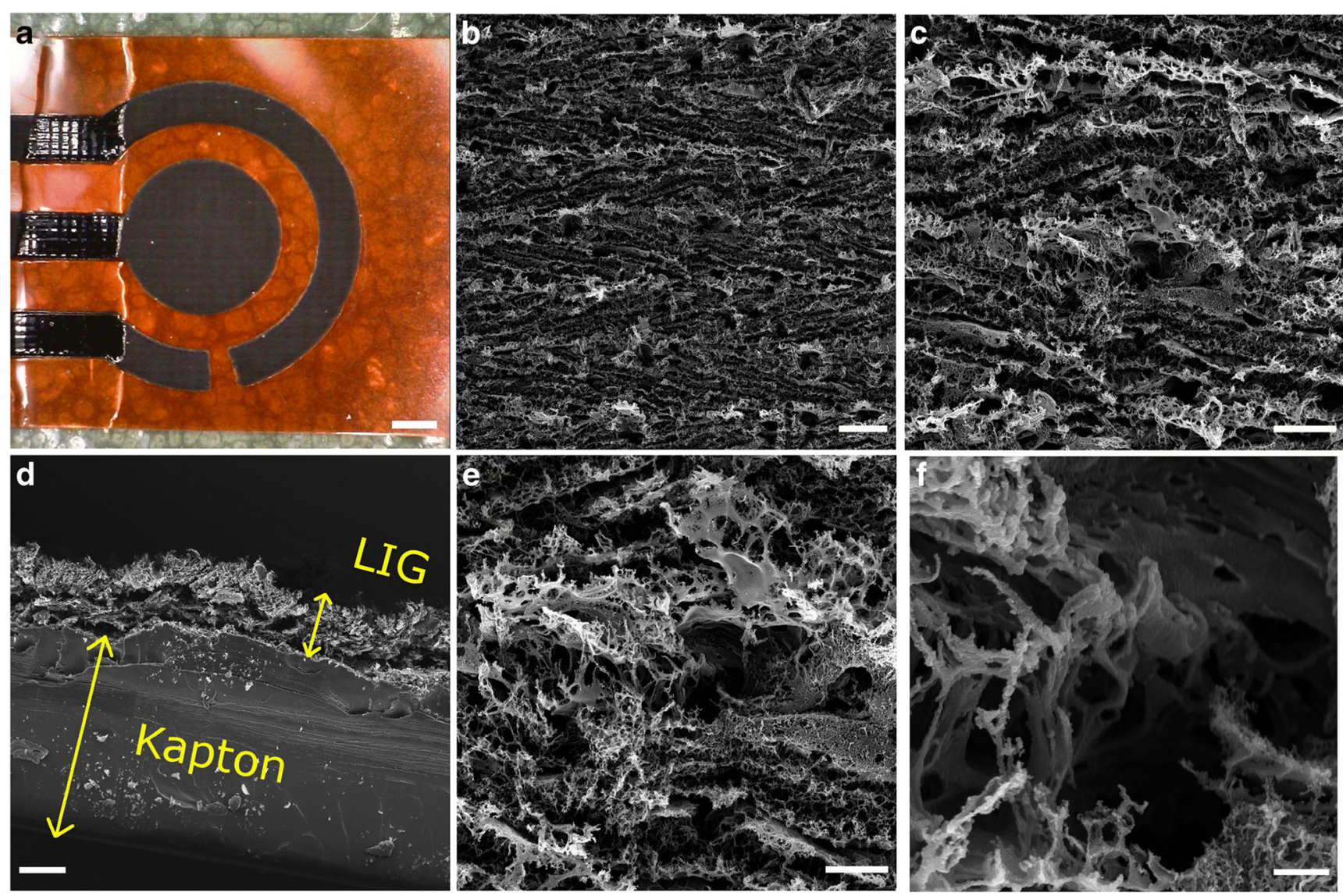

Fig. 6 Photograph and SEM micrographs of LIG $(1 / 10 / 1000 \times 1000)$ scale bars in pictures a-f are 500, 20, 10, 20, 5, and $1 \mu \mathrm{m}$

was irradiated an average number of 18 times because of laser beam overlap in $x$ - and $y$-directions (see also Fig. S8).

As seen in the cross-sectional view in Fig. 6d, a significant part of the Kapton substrate, around $100 \mu \mathrm{m}$, remains intact after lasing and serves as a support for the more delicate coralreef-like LIG structure which has an average height of $27 \pm$ $3 \mu \mathrm{m}$ as determined by SEM.

SEM pictures of LIG obtained at other scribing conditions revealed significantly different morphologies. This observation has already been disclosed by others, e.g., Tiliakos et al. identified five different morphic groups of LIG with differences in Raman spectra, electrical conductivity, and wettability [8]. They used a galvanometric laser processing unit which permitted a high degree of variation in scan rate and pulse frequency. On the other hand, Duy et al. reported the creation of long LIG-fibers (LIGF), by decreasing the pulse frequency while at the same time using a smaller beam diameter, effectively reducing the beam overlap [26]. The commercial flatbed laser processing unit used by their group is similar to the one in this study and we obtained LIGF by reducing pulse densities to 500 PPI but still using a beam diameter of $125 \mu \mathrm{m}$ (Fig. S12). Apparently, the fibrous structure was also created when a certain beam overlap occurs, in this case approx. 4 times. In fact, even at $1000 \times 1000$ PPI, brush-like LIG structures could be observed, given the right power/ speed combination, as seen in Fig. $4 \mathrm{f}$ above. Correlating the morphology to the electroanalytical performance in Fig. 4e, it can be deduced that large brushlike structures are not favorable for electroanalysis as likely looser structures lead to a higher resistance and hence worse electroanalytical performance. A possible gain in overall surface area hence does not translate here into better electrodes for analysis.

The electrochemically active surface area (ESA) of LIG electrodes, determined with the voltammetric method via the Randles-Sevcik equation, was $0.107 \mathrm{~cm}^{2}$ (about 1.8 times the geometrical surface area $A_{\mathrm{GEO}}$, Fig. $7 \mathrm{~b}$ ) and the calculated effective heterogeneous electron transfer coefficient $\left(k^{0, \text { eff }}\right)$ for the $\left[\mathrm{Fe}(\mathrm{CN})_{6}\right]^{3-/ 4-}$ couple was $0.003 \mathrm{~cm} \mathrm{~s}^{-1}$. For comparison, commercial screen-printed carbon electrodes exhibited a lower calculated ESA of 0.9 times $A_{\mathrm{GEO}}$ and a comparable $k^{0, \text { eff }}$ of $0.002 \mathrm{~cm} \mathrm{~s}^{-1}$. However, the Randles-Sevcik relationship-like the Nicholson method for the determination of $k^{0 \text {,eff }}$-is only strictly applicable to smooth electrode surfaces with planar semi-infinite diffusion of dissolved redox species. Therefore, the values of 


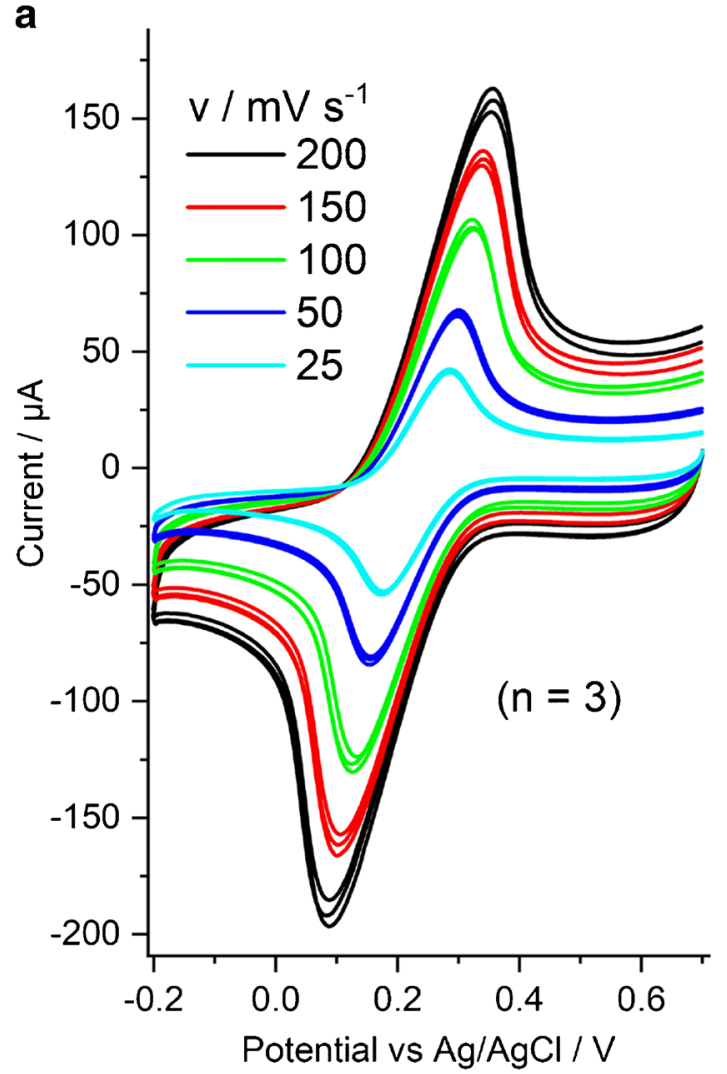

Fig. 7 a $\mathrm{CV}$ in $5 \mathrm{mM} \mathrm{K}_{3}\left[\mathrm{Fe}(\mathrm{CN})_{6}\right]$ in $0.1 \mathrm{M} \mathrm{KCl}$ on $1 / 10 / 1000 \times 1000$ electrodes at various scan rates. b Plot of cathodic peak current vs $v^{1 / 2}$. The slope was divided by the factors in the Randles-Sevcik equation $\left(0.4463 \mathrm{~F}^{3 / 2}(D / R T)^{1 / 2} \mathrm{C}\right)$, with $F=96,485 \mathrm{~A} \mathrm{~s} \mathrm{~mol}^{-1}, D=7.63 \mathrm{E}$

ESA and $k^{0, \text { eff }}$ reported here should be regarded as an estimate rather than accurate. The specific surface area of LIG $1 / 10 / 1000 \times 1000$ determined by nitrogen adsorption isotherm analysis was approx. 330 times the geometrical surface area. Obviously, this does not correlate to the determined ESA. We assume that the electrochemical experiment solution may not enter all of the pores and cavities due to hydrophobic pouches and that some areas are not b
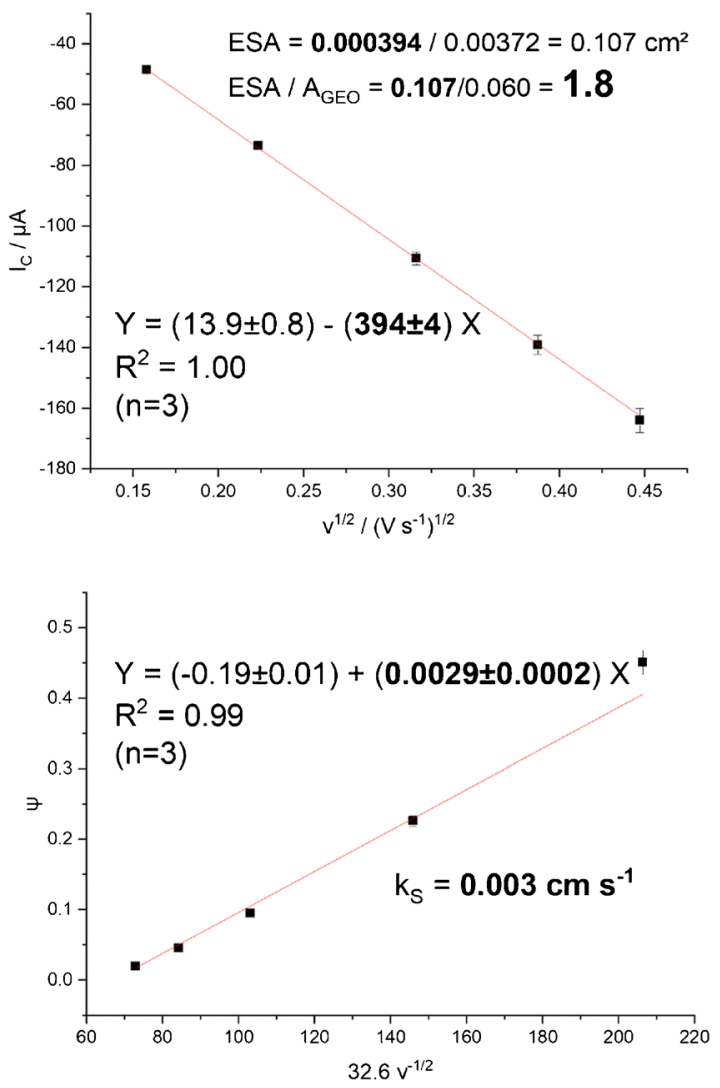

$-6 \mathrm{~cm}^{2} \mathrm{~s}^{-1}, R=8.314 \mathrm{~J} \mathrm{~K}^{-1} \mathrm{~mol}^{-1}, T=296.15 \mathrm{~K}, C=5 \mathrm{E}-6 \mathrm{~mol} \mathrm{~cm}{ }^{-3}$, to yield $0.107 \mathrm{~cm}^{2}$. Division by the geometric surface of $0.06 \mathrm{~cm}^{2}$ yielded a ratio of 1.8. $\mathbf{c}$ Nicholson plot based on the same data: slope $=k^{0, \text { eff }}=$ $0.003 \mathrm{~cm} \mathrm{~s}^{-1}$. 3-electrode design LIG electrodes were used

conductively connected well enough, which leads to this dramatic difference in measurements.

Table 1 compares the values of ESA, $k^{0, \text { eff }}$, and sheet resistance to previously published data for LIG or very similar material (LSG). The values we report here are in the spectrum of previously reported results.

Finally, electrochemical impedance spectroscopy revealed extremely low impedance values compared to commercial

Table 1 Electrode material characteristics compared to previous publications of similar materials (NA, not available; ESA and electron transfer rate coefficient were in all cases determined with the voltammetric method)

\begin{tabular}{|c|c|c|c|c|c|c|}
\hline Laser & Substrate & $A_{\mathrm{GEO}}\left(\mathrm{cm}^{2}\right)$ & $\begin{array}{l}\text { Roughness } \\
\text { factor } \\
\left(\mathrm{ESA} / \mathrm{A}_{\mathrm{GEO}}\right)\end{array}$ & $\begin{array}{l}\text { Sheet } \\
\text { resistance } \\
\left(\Omega \mathrm{sq}^{-1}\right)\end{array}$ & $k^{0, \text { eff }}\left(\mathrm{cm} \mathrm{s}^{-1}\right)$ & Ref. \\
\hline $788 \mathrm{~nm}$ diode & Graphene oxide & 0.16 & NA & 80 & 0.0133 & [27] \\
\hline $788 \mathrm{~nm}$ diode & Graphene oxide & 0.07 & NA & 589 & 0.0237 & {$[28]$} \\
\hline $10.6 \mu \mathrm{m}, \mathrm{CO}_{2}$ & Kapton & 0.07 & 1.8 & NA & 0.0044 & {$[15]$} \\
\hline $405 \mathrm{~nm}$ diode & Kapton & 0.13 & 2.7 & NA & 0.0030 & [18] \\
\hline $10.6 \mu \mathrm{m}, \mathrm{CO}_{2}$ & Kapton & 0.07 & 1.5 & 12,700 & 0.0146 & [29] \\
\hline $10.6 \mu \mathrm{m}, \mathrm{CO}_{2}$ & Lignin film & 0.07 & 1.8 & 2.8 & 0.0090 & {$[30]$} \\
\hline $10.6 \mu \mathrm{m}, \mathrm{CO}_{2}$ & Kapton & 0.06 & 1.8 & 23 & 0.0030 & This work \\
\hline
\end{tabular}


screen-printed carbon electrodes of the same geometrical size (Fig. S13) We conceive that especially this characteristic will
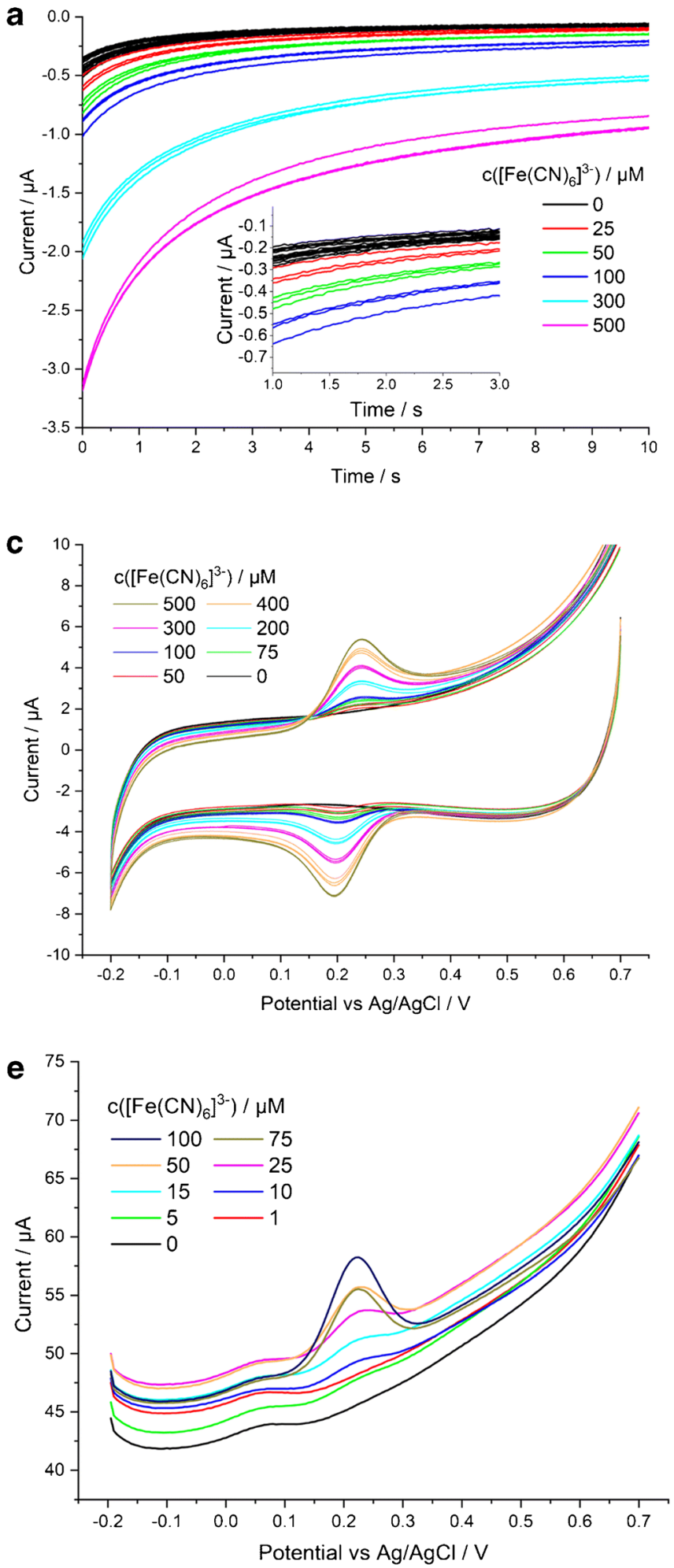

Fig. 8 Dose-response experiments to $\left[\mathrm{Fe}(\mathrm{CN})_{6}\right]^{3-}$ on LIG electrodes $(1 / 10 / 1000 \times 1000)$ using chronoamperometry $(\mathbf{a}+\mathbf{b}$, step from $0.4 \mathrm{~V}$ to $0.0 \mathrm{~V})$, cyclic voltammetry $(\mathbf{c}+\mathbf{d})$, and square-wave voltammetry $(\mathbf{e}+$ f). Each electrode was used for only a single measurement and three make LIG an interesting material for EIS sensors as also demonstrated by us and other groups previously [29, 31, 32].
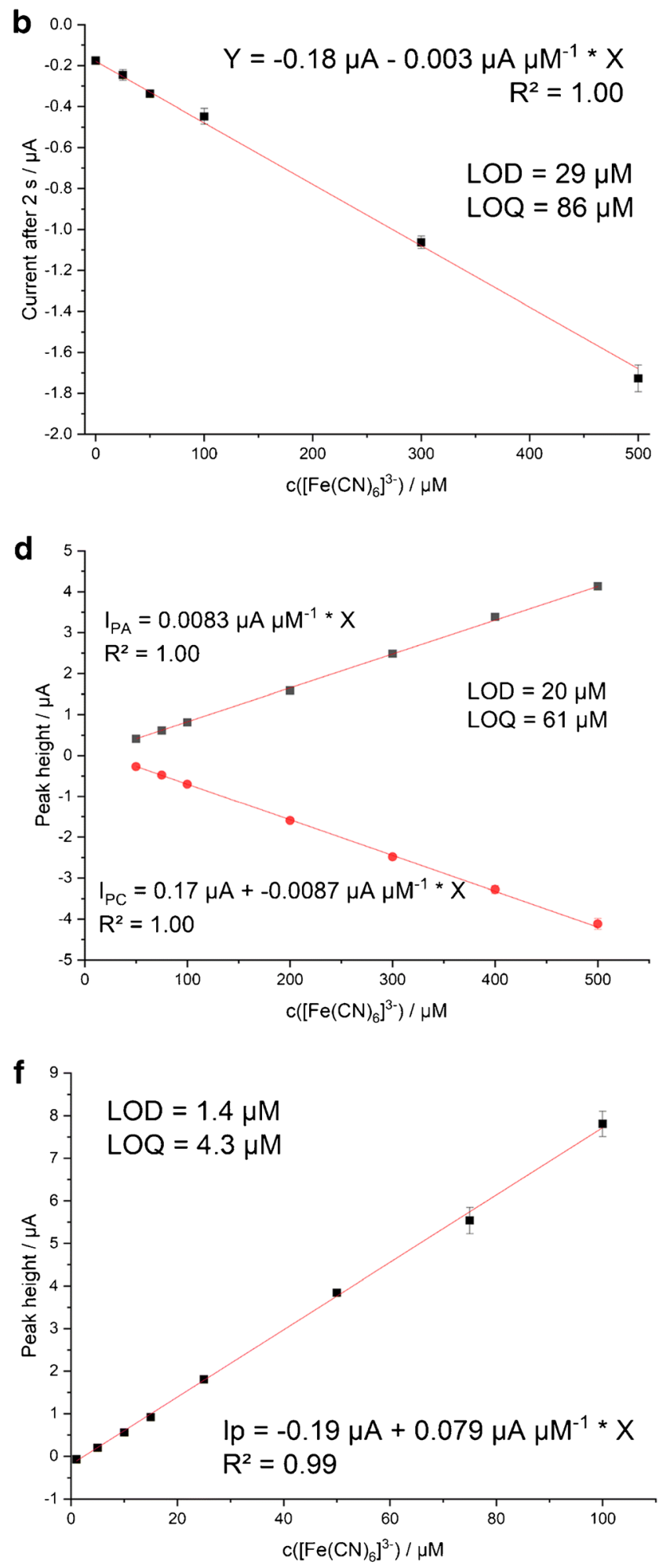

electrodes were used per concentration level. In SWV, each fresh electrode displayed a slightly different background current and so, for clarity, each concentration level in $\mathbf{e}$ is represented by one voltammetric curve only. 3-electrode design LIG electrodes were used 


\section{Voltammetric applications of LIG electrodes}

The electrochemical behavior of various molecules of interest on LIG electrodes was investigated by CV to test the broad applicability of this electrode type for chemical sensing (Fig. S15). We observed that $\left[\mathrm{Ru}\left(\mathrm{NH}_{3}\right)_{6}\right]^{3+}$, a representative for molecules that undergo outer-sphere electron transfer, was just as easily detected as $\left[\mathrm{Fe}(\mathrm{CN})_{6}\right]^{3-}$, which is classified as a more surface-dependent redox species (Fig. S15A) [33]. The detection of dopamine (DA) can generally be hindered by the presence of ascorbic acid (AA) or uric acid (UA) which oxidize at very similar potentials. In Fig. S15B, the peaks of DA, AA, and UA are sufficiently separated on LIG as also shown by our collaborators earlier [14]. This beneficial effect might be partly explained by the transition from a planar semi-infinite diffusion regime to thinlayer diffusion, as Compton's group has pointed out in the past about the modification of glassy carbon electrodes with nanomaterials [34]. Figure S15C and D demonstrate the detection of p-nitrophenol and paracetamol, which could be direct analytes of interest, while a $\mathrm{CV}$ of the common redox mediator methylene blue (MB) is shown in Fig. S15E. MB adsorbs easily onto LIG, as indicated by the very low peak separation and increased currents in consecutive scans and, in fact, Rathinam et al. already suggested LIG powder as adsorbent for MB in water treatment [35]. Since MB is also used as an electron mediator in biosensors, the strong adsorption may be beneficial in that case.

Generally, an overall high background capacitance due to the large inherent surface area can be observed on LIG electrodes, paired with an excellent ability for oxidation and reduction reactions of inner and outer-sphere electroactive species. We observed that the current response of different redox species was significantly larger on LIG compared to commercial screen-printed carbon or glassy carbon electrodes. This may be caused by not only the porous nature of LIG but also the apparent presence of many reactive edge sites (see the large $D$ peak in the Raman spectrum of Fig. S6) which may cause this improved electrocatalytic activity [36]. This demonstrates the overall utility of LIG as sensitive electrode material for analytical applications.

Finally, we compared the detection of $\left[\mathrm{Fe}(\mathrm{CN})_{6}\right]^{3-}$ on the chosen LIG electrode type using chronoamperometry (CA), cyclic voltammetry (CV), and square-wave voltammetry (SWV). Detection via CV below a concentration of $50 \mu \mathrm{M}$ was not possible (Fig. 8c, d), the lowest detectable concentrations with $\mathrm{CA}$ and SWV were $25 \mu \mathrm{M}$ and $5 \mu \mathrm{M}$ respectively. Upwards, the response was linear to the highest tested concentration of $500 \mu \mathrm{M}$ with $\mathrm{CA}$ and $\mathrm{CV}$, while SWV allowed a linear calibration up to $100 \mu \mathrm{M}$ with signals increasing less at higher concentrations (only linear part of calibration displayed in Fig. 8f).
The SWV calibration of $\mathrm{K}_{3}\left[\mathrm{Fe}(\mathrm{CN})_{6}\right]$ on LIG electrodes in Fig. 8e, f can be compared to data from commercially available screen-printed carbon electrodes (Dropsens, DRP$110)$ in Fig. S14. Our LIG electrodes express a similar limit of detection (LOD and LOQ with SPE $=1.0$ and $3.0 \mu \mathrm{M})$ although the screen-printed electrodes show less background current.

\section{Conclusion}

Laser-induced graphene (LIG) was investigated with focus on understanding the effect fabrication parameters and morphologies have on the electroanalytical performance. It was found that the material properties can be tuned in the production process, and, in fact, a large range of workable parameters was found, which provides good leverage for large-scale, maximum productivity at sufficient electrode performance, tailored toward the desired application. The best analytical electrodes were obtained at low, frequent energy input, i.e., low laser power, low scanning speed, and high spatial pulse density. Key features of the material are its large porosity, sufficient mechanical stability, and chemical purity as no binder substances are required such as in screen-printed graphite electrodes. Thorough electroanalytical characterization and application to a variety of analyte molecules reveal LIG to serve well for detection in the low micromolar range and provide reliable data. As to be expected for a crystalline carbon material, some chemical species irreversibly and readily adsorbed onto LIG, which suggests its strength for single-use electrode systems. This works well with most healthcare applications, where the single-use approach dominates the diagnostic market. The nature of the laser patterning process poses two main constraints on LIG: the features cannot be much smaller than $100 \mu \mathrm{m}$ and the resulting material is always porous and never flat. Although recently, a group reported LIG with a slightly higher resolution of $50 \mu \mathrm{m}$ by switching the IR-laser for UV [37], a much lower feature size on the scale of a few $\mu \mathrm{m}$ or even nanometers, as achieved by lithography, is generally impossible for LIG. It is therefore not suited to create nanoscale interdigitated arrays, e.g., for detection strategies using redox cycling [38]. However, the resolution is comparable to screen-printing and LIG is very well suitable for general use [39].

A perceivable drawback of the binder-free LIG electrode material is its lower mechanical stability. For example, the highest conductive LIG cannot be used as an electrode as it lifts off its substrate when slightly bent. It has been proposed to render LIG mechanically more robust by infusion of silicon [40, 41] or cement [42], but 
electrode surface area and superior electron transfer processes suffer from this. Thus, instead, it is proposed that a balance between optimal conductivity and mechanical robustness needs to be found and tailored toward the final application.

Supplementary Information The online version contains supplementary material available at https://doi.org/10.1007/s00604-021-04792-3.

Acknowledgements We would like to thank Marcel Simsek for the SEM pictures, Yongjiu Liu and Husam Alshareef for their help with the XPS analysis, Synthia Ndeta for CV data on methylene blue, and Rainer Müller for the nitrogen adsorption measurements.

Funding Open Access funding enabled and organized by Projekt DEAL. This work was supported in part by funding from King Abdullah University of Science and Technology (KAUST).

\section{Declarations}

Conflict of interest The authors declare no competing interests.

Open Access This article is licensed under a Creative Commons Attribution 4.0 International License, which permits use, sharing, adaptation, distribution and reproduction in any medium or format, as long as you give appropriate credit to the original author(s) and the source, provide a link to the Creative Commons licence, and indicate if changes were made. The images or other third party material in this article are included in the article's Creative Commons licence, unless indicated otherwise in a credit line to the material. If material is not included in the article's Creative Commons licence and your intended use is not permitted by statutory regulation or exceeds the permitted use, you will need to obtain permission directly from the copyright holder. To view a copy of this licence, visit http://creativecommons.org/licenses/by/4.0/.

\section{References}

1. Heller A, Feldman B (2008) Electrochemical glucose sensors and their applications in diabetes management. Chem Rev 108:24822505. https://doi.org/10.1021/cr068069y

2. Newman JD, Turner APF (2005) Home blood glucose biosensors: a commercial perspective. Biosens Bioelectron 20:2435-2453. https://doi.org/10.1016/j.bios.2004.11.012

3. Taleat Z, Khoshroo A, Mazloum-Ardakani M (2014) Screenprinted electrodes for biosensing: a review (2008-2013). Microchim Acta 181:865-891. https://doi.org/10.1007/s00604014-1181-1

4. da Silva ETSG, Souto DEP, Barragan JTC et al (2017) Electrochemical biosensors in point-of-care devices: recent advances and future trends. ChemElectroChem 4:778-794. https:// doi.org/10.1002/celc.201600758

5. Barton J, García MBG, Santos DH, Fanjul-Bolado P, Ribotti A, McCaul M, Diamond D, Magni P (2016) Screen-printed electrodes for environmental monitoring of heavy metal ions: a review. Microchim Acta 183:503-517. https://doi.org/10.1007/s00604015-1651-0

6. Lin J, Peng Z, Liu Y, Ruiz-Zepeda F, Ye R, Samuel ELG, Yacaman MJ, Yakobson BI, Tour JM (2014) Laser-induced porous graphene films from commercial polymers. Nat Commun 5:5714
7. Kurra N, Jiang Q, Nayak P, Alshareef HN (2019) Laser-derived graphene: a three-dimensional printed graphene electrode and its emerging applications. Nano Today 24:81-102. https://doi.org/10. 1016/j.nantod.2018.12.003

8. Tiliakos A, Ceaus C, Iordache SM, Vasile E, Stamatin I (2016) Morphic transitions of nanocarbons via laser pyrolysis of polyimide films. J Anal Appl Pyrolysis 121:275-286. https://doi.org/10.1016/ j.jaap.2016.08.007

9. Ye R, James DK, Tour JM (2018) Laser-induced graphene: from discovery to translation. Adv Mater 0:1803621. https://doi.org/10. 1002/adma.201803621, 31

10. Li L, Zhang J, Peng Z, Li Y, Gao C, Ji Y, Ye R, Kim ND, Zhong Q, Yang Y, Fei H, Ruan G, Tour JM (2016) High-performance pseudocapacitive microsupercapacitors from laser-induced graphene. Adv Mater 28:838-845. https://doi.org/10.1002/adma. 201503333

11. Ye R, Chyan Y, Zhang J et al (2017) Laser-induced graphene formation on wood. Adv Mater 29:1702211-n/a. https://doi.org/10. 1002/adma.201702211

12. Li Y, Luong DX, Zhang J et al (2017) Laser-induced graphene in controlled atmospheres: from superhydrophilic to superhydrophobic surfaces. Adv Mater 29:1700496-n/a. https:// doi.org/10.1002/adma.201700496

13. Ye R, James DK, Tour JM (2018) Laser-induced graphene. Acc Chem Res 51:1609-1620. https://doi.org/10.1021/acs.accounts. 8 b00084

14. Nayak P, Kurra N, Xia C, Alshareef HN (2016) Highly efficient laser scribed graphene electrodes for on-chip electrochemical sensing applications. Adv Electron Mater 2:1600185. https://doi.org/10. 1002/aelm.201600185

15. Fenzl C, Nayak P, Hirsch T, Wolfbeis OS, Alshareef HN, Baeumner AJ (2017) Laser-scribed graphene electrodes for aptamer-based biosensing. ACS Sens 2:616-620. https://doi.org/ 10.1021/acssensors.7b00066

16. Bauer M, Wunderlich L, Weinzierl F, Lei Y, Duerkop A, Alshareef HN, Baeumner AJ (2020) Electrochemical multi-analyte point-ofcare perspiration sensors using on- chip three-dimensional graphene electrodes. Anal Bioanal Chem 413:763-777. https://doi.org/10. 1007/s00216-020-02939-4

17. Tehrani F, Bavarian B (2016) Facile and scalable disposable sensor based on laser engraved graphene for electrochemical detection of glucose. Sci Rep 6:27975

18. Vanegas D, Patiño L, Mendez C et al (2018) Laser scribed graphene biosensor for detection of biogenic amines in food samples using locally sourced materials. Biosensors:8. https://doi.org/10.3390/ bios 8020042

19. Garland NT, McLamore ES, Cavallaro ND et al (2018) Flexible laser-induced graphene for nitrogen sensing in soil. ACS Appl Mater Interfaces 10:39124-39133. https://doi.org/10.1021/acsami. 8 b10991

20. Kucherenko IS, Sanborn D, Chen B, Garland N, Serhan M, Forzani E, Gomes C, Claussen JC (2020) Ion-selective sensors based on laser-induced graphene for evaluating human hydration levels using urine samples. Adv Mater Technol 5:1901037. https://doi.org/10. 1002/admt.201901037

21. Cardoso AR, Marques AC, Santos L, Carvalho AF, Costa FM, Martins R, Sales MGF, Fortunato E (2019) Molecularly-imprinted chloramphenicol sensor with laser-induced graphene electrodes. Biosens Bioelectron 124-125:167-175. https://doi.org/10.1016/j. bios.2018.10.015

22. Beduk T, Ait Lahcen A, Tashkandi N, Salama KN (2020) One-step electrosynthesized molecularly imprinted polymer on laser scribed graphene bisphenol a sensor. Sensors Actuators B Chem 314: 128026. https://doi.org/10.1016/j.snb.2020.128026

23. Lahcen AA, Rauf S, Beduk T, Durmus C, Aljedaibi A, Timur S, Alshareef HN, Amine A, Wolfbeis OS, Salama KN (2020) 
Electrochemical sensors and biosensors using laser-derived graphene: a comprehensive review. Biosens Bioelectron: 112565 . https://doi.org/10.1016/j.bios.2020.112565

24. Susi T, Ayala P (2015) Doping carbon nanomaterials with heteroatoms. In: Carbon Nanomaterials for advanced energy systems. John Wiley \& Sons, Ltd, pp 133-161

25. Mamleyev ER, Heissler S, Nefedov A, Weidler PG, Nordin N, Kudryashov VV, Länge K, MacKinnon N, Sharma S (2019) Laser-induced hierarchical carbon patterns on polyimide substrates for flexible urea sensors. Npj Flex Electron 3:2. https://doi.org/10. 1038/s41528-018-0047-8

26. Duy LX, Peng Z, Li Y, Zhang J, Ji Y, Tour JM (2018) Laserinduced graphene fibers. Carbon 126:472-479. https://doi.org/10. 1016/j.carbon.2017.10.036

27. Strong V, Dubin S, El-Kady MF et al (2012) Patterning and electronic tuning of laser scribed graphene for flexible all-carbon devices. ACS Nano 6:1395-1403. https://doi.org/10.1021/ nn204200w

28. Griffiths K, Dale C, Hedley J, Kowal MD, Kaner RB, Keegan N (2014) Laser-scribed graphene presents an opportunity to print a new generation of disposable electrochemical sensors. Nanoscale 6:13613-13622. https://doi.org/10.1039/C4NR04221B

29. Soares RRA, Hjort RG, Pola CC, Parate K, Reis EL, Soares NFF, McLamore ES, Claussen JC, Gomes CL (2020) Laser-induced graphene electrochemical immunosensors for rapid and label-free monitoring of Salmonella enterica in chicken broth. ACS Sens 5: 1900-1911. https://doi.org/10.1021/acssensors.9b02345

30. Lei Y, Alshareef AH, Zhao W, Inal S (2020) Laser-scribed graphene electrodes derived from lignin for biochemical sensing. ACS Appl Nano Mater 3:1166-1174. https://doi.org/10.1021/ acsanm.9b01795

31. Yagati AK, Behrent A, Beck S, Rink S, Goepferich AM, Min J, Lee MH, Baeumner AJ (2020) Laser-induced graphene interdigitated electrodes for label-free or nanolabel-enhanced highly sensitive capacitive aptamer-based biosensors. Biosens Bioelectron 164: 112272. https://doi.org/10.1016/j.bios.2020.112272

32. Puetz P, Behrent A, Baeumner AJ, Wegener J (2020) Laser-scribed graphene (LSG) as new electrode material for impedance-based cellular assays. Sensors Actuators B Chem 321:128443. https:// doi.org/10.1016/j.snb.2020.128443

33. McCreery RL (2008) Advanced carbon electrode materials for molecular electrochemistry. Chem Rev 108:2646-2687. https://doi. org $/ 10.1021 / \mathrm{cr} 068076 \mathrm{~m}$
34. Henstridge MC, Dickinson EJF, Aslanoglu M, Batchelor-McAuley C, Compton RG (2010) Voltammetric selectivity conferred by the modification of electrodes using conductive porous layers or films: the oxidation of dopamine on glassy carbon electrodes modified with multiwalled carbon nanotubes. Sensors Actuators B Chem 145:417-427. https://doi.org/10.1016/j.snb.2009.12.046

35. Rathinam K, Singh SP, Li Y, Kasher R, Tour JM, Arnusch CJ (2017) Polyimide derived laser-induced graphene as adsorbent for cationic and anionic dyes. Carbon 124:515-524. https://doi.org/10. 1016/j.carbon.2017.08.079

36. Brownson DAC, Varey SA, Hussain F, Haigh SJ, Banks CE (2014) Electrochemical properties of CVD grown pristine graphene: monolayer- vs. quasi-graphene. Nanoscale 6:1607-1621. https:// doi.org/10.1039/C3NR05643K

37. Carvalho AF, Fernandes AJS, Leitão C, Deuermeier J, Marques AC, Martins R, Fortunato E, Costa FM (2018) Laser-induced graphene strain sensors produced by ultraviolet irradiation of polyimide. Adv Funct Mater 28:1805271. https://doi.org/10.1002/adfm. 201805271

38. Alayo N, Fernández-Sánchez C, Baldi A, Esquivel JP, Borrisé X, Pérez-Murano F (2016) Gold interdigitated nanoelectrodes as a sensitive analytical tool for selective detection of electroactive species via redox cycling. Microchim Acta 183:1633-1639. https://doi. org/10.1007/s00604-016-1792-9

39. Vasudivan S, Zhiping W (2010) Fine line screen printed electrodes for polymer microfluidics. 2010 12th Electronics Packaging Technology Conference. pp 89-93. https://doi.org/10.1109/EPTC. 2010.5702612

40. Hui X, Xuan X, Kim J, Park JY (2019) A highly flexible and selective dopamine sensor based on Pt-Au nanoparticle-modified laser-induced graphene. Electrochim Acta 328:135066. https://doi. org/10.1016/j.electacta.2019.135066

41. Parmeggiani M, Zaccagnini P, Stassi S, Fontana M, Bianco S, Nicosia C, Pirri CF, Lamberti A (2019) PDMS/polyimide composite as an elastomeric substrate for multifunctional laser-induced graphene electrodes. ACS Appl Mater Interfaces 11:3322133230. https://doi.org/10.1021/acsami.9b10408

42. Stanford MG, Yang K, Chyan Y, Kittrell C, Tour JM (2019) Laserinduced graphene for flexible and embeddable gas sensors. ACS Nano 13:3474-3482. https://doi.org/10.1021/acsnano.8b09622

Publisher's note Springer Nature remains neutral with regard to jurisdictional claims in published maps and institutional affiliations. 